\title{
FLIGHT DYNAMICS ANALYSIS AND CONTROL OF TRANSPORT AIRCRAFT SUBJECT TO FAILURE
}

\author{
O. Daşkıran ${ }^{1}$ and M. Ş. Kavsaoğlu ${ }^{2}$ \\ ${ }^{1}$ Istanbul Technical University \\ Department of Aeronautical Engineering \\ Istanbul 34469, Turkey \\ ${ }^{2}$ Anadolu University \\ Faculty of Aeronautics and Astronautics \\ Eskisehir 26470, Turkey
}

\begin{abstract}
After a structural damage or component failure during any flight mode, aircraft dynamics are dramatically altered. A quick and adequate stabilization effort is crucial. Flight dynamics for several failure scenarios are analyzed. Necessary amounts of control deflections for postfailure trim are calculated. These trim values are used as control input in an open loop manner and validity of this approach is tested via flight simulations. Alternatively, a closed loop flight control system, which does not need the postfailure trim values, is also designed. This closed loop controller is based on a linearized aircraft model whereas flight simulations are based on nonlinear aircraft dynamics.
\end{abstract}

\section{NOMENCLATURE}

$\begin{array}{ll}\text { AR } & \text { aspect ratio } \\ b & \text { span length, m } \\ \bar{c} & \text { mean aerodynamic chord, } \mathrm{m} \\ D & \text { drag, } \mathrm{N} \\ F_{A x}, F_{A y}, F_{A z} & \text { aerodynamic force components } \\ F_{T x}, F_{T y}, F_{T z} & \text { thrust force components } \\ g & \text { acceleration of gravity, } \mathrm{m} / \mathrm{s}^{2} \\ i_{h} & \text { stabilizer incidence angle } \\ I_{x x}, I_{y y}, I_{z z} & \text { moments of inertia, } \mathrm{kg} \cdot \mathrm{m}^{2} \\ I_{x y}, I_{y z}, I_{x z} & \text { products of inertia, } \mathrm{kg} \cdot \mathrm{m}^{2} \\ L & \text { lift, } \mathrm{N} \\ L_{A}, L_{T} & \text { aerodynamic and thrust induced rolling moments, } \mathrm{N} \cdot \mathrm{m}\end{array}$




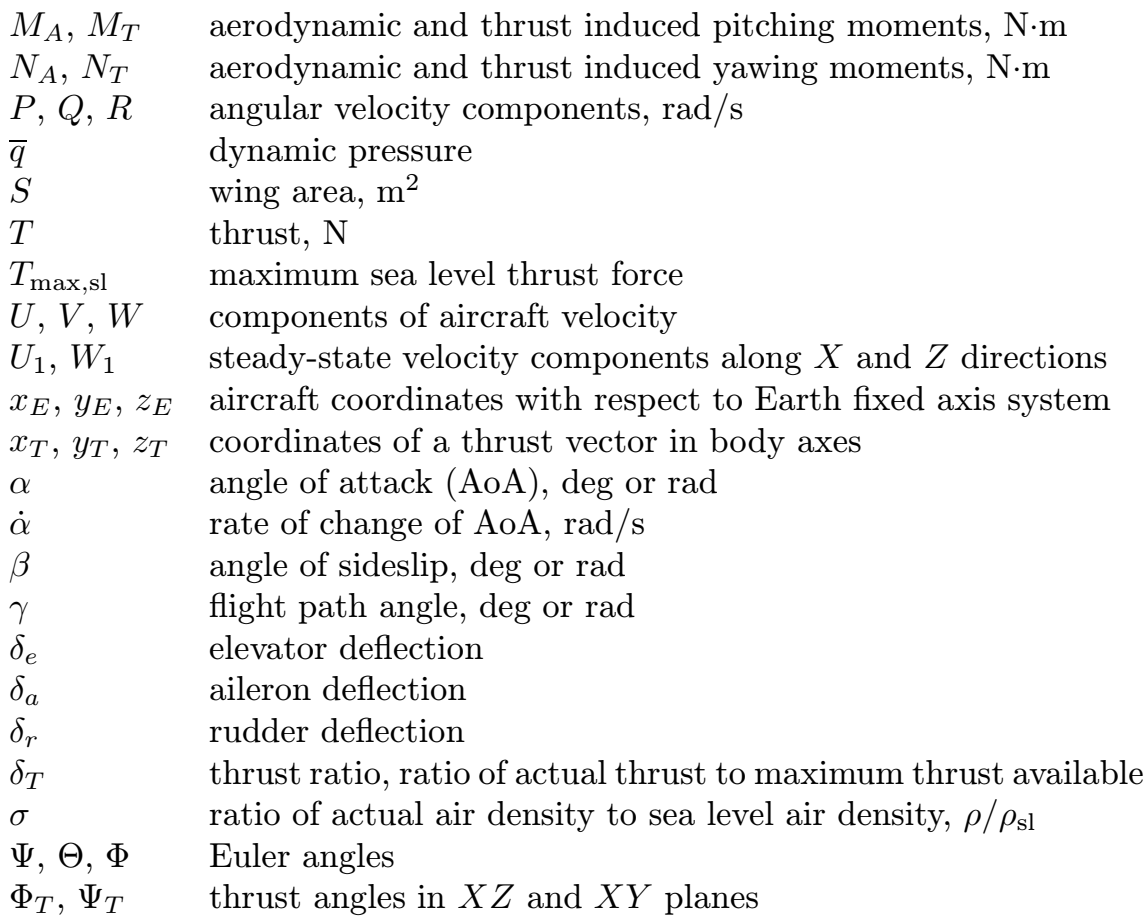

\section{INTRODUCTION}

Failure or damage of components during flight is an important aspect that affects both lateral and longitudinal dynamics of aircraft dramatically. Engine thrust loss, control surface damages, and actuator failures generate large amount of irregular forces and moments which lead to harmful consequences. Several studies have been conducted on this subject.

For the engine out case, McLean [1] discusses control augmentation for partial thrust loss of general aviation aircraft during takeoff and landing. Magni et al. [2] suggest a robust autopilot design for the problem. Kordt and Ackermann [3] use parameter uncertainty methods to alleviate uncertain loads on a commercial aircraft, comparing the results with human pilot interference. A more recent flight dynamics study performed by Özdemir and Kavsaoğlu simulates the event with precalculated open loop control [4]. This approach defines failure as one engine full thrust loss and introduces engine wind milling drag due to inoperative jet engine. This reduces the amount of uncertainties and enables a more realistic linear model defining the event. Stabilization after damage on aerodynamic and control surfaces is also equally important but more difficult as the aerodynamic 
model is altered and large amount of uncertainties are introduced. In the literature, there are many studies on detecting and compensating damage. Shtessel [5] and Hess et al. [6] use sliding mode controllers to track pilots input on a damaged military jet aircraft. Boskovic et al. [7] use multimodel switching control theory to model each failure differently. Bodson and Groszkiewicz [8] and Liu et al. [9] make use of adaptive control schemes for actuator failures. Mack et al. [10] suggest a model reference adaptive damage detector by comparing the damaged responses to healthy model.

Postfailure trim conditions and flight dynamics for one engine thrust loss and rudder actuator failure cases are investigated. An open loop controller using this trim values is employed. A new closed loop autopilot is also designed, with modern control synthesis techniques, to hold the aircraft attitude and position close to the cruise conditions before the failures. Nonlinear flight simulations are performed and results are compared with open loop case.

\section{EQUATIONS OF MOTION}

The nonlinear force and moment equations for 6 degrees of freedom aircraft model can be written in body axis by 3 forces, 3 moments, 3 kinematic, and 3 linear position equations [11]:

$$
\begin{array}{r}
m(\dot{U}+Q W-R V)=F_{G x}+F_{A x}+F_{T x}=F_{x} ; \\
m(\dot{V}+R U-P W)=F_{G y}+F_{A y}+F_{T y}=F_{y} ; \\
m(\dot{W}+P V-Q U)=F_{G z}+F_{A z}+F_{T z}=F_{z} ; \\
\dot{P} I_{x x}+Q R\left(I_{z z}-I_{y y}\right)-(\dot{R}+P Q) I_{x z}=L_{A}+L_{T}=L ; \\
\dot{Q} I_{y y}+P R\left(I_{z z}-I_{x x}\right)+\left(P^{2}-R^{2}\right) I_{x z}=M_{A}+M_{T}=M ; \\
\dot{R} I_{z z}+P Q\left(I_{y y}-I_{x x}\right)+(Q R-\dot{P}) I_{x z}=N_{A}+N_{T}=N
\end{array}
$$

where $U, V$, and $W$ are the velocities; and $P, Q$, and $R$ are the angular rates on body axis. Aircraft attitude by means of Euler angles and displacement with respect to Earth frame can be given as a function of these variables [11,12]:

$$
\begin{aligned}
\dot{\phi} & =P+\frac{R \cos \Phi+Q \sin \Phi}{\cos \Theta} \sin \Theta \\
\dot{\theta} & =Q \cos \Phi-R \sin \Phi \\
\dot{\psi} & =\frac{R \cos \Phi+Q \sin \Phi}{\cos \Theta} ; \\
{\left[\begin{array}{c}
\dot{x}_{E} \\
\dot{y}_{E} \\
\dot{z}_{E}
\end{array}\right] } & =\left[\begin{array}{ccc}
\cos \Psi-\sin \Psi & 0 \\
\sin \Psi & \cos \Psi & 0 \\
0 & 0 & 1
\end{array}\right]\left[\begin{array}{ccc}
\cos \Theta & 0 & \sin \Theta \\
0 & 1 & 0 \\
-\sin \Theta & 0 & \cos \Theta
\end{array}\right]\left[\begin{array}{ccc}
1 & 0 & 0 \\
0 & \cos \Phi-\sin \Phi \\
0 & \sin \Phi & \cos \Phi
\end{array}\right]\left[\begin{array}{c}
U \\
V \\
W
\end{array}\right] .
\end{aligned}
$$


Indices $G, A$, and $T$ represent gravity, aerodynamic and thrust contributions, respectively. Gravity forces on body axis are the functions of Euler angles and weight of the aircraft:

$$
F_{G x}=-m g \sin \theta ; \quad F_{G y}=m g \sin \phi \cos \theta ; \quad F_{G z}=m g \cos \phi \cos \theta .
$$

Aerodynamic forces and moments are the functions of wing geometry $(\bar{c}, \bar{b}$, and $S$ ), dynamic pressure $(\bar{q})$, and dimensionless aerodynamic coefficients derived by the AAA ${ }^{\circledR}$ program [13]. These coefficients constitute a linear aerodynamic model. Equations also contain unsteady $\dot{\alpha}, \dot{\beta}, p, Q$, and $r$ terms which are zero at steady modes as cruise, although they can be very effective on aircraft dynamics.

Aerodynamic forces lift, $L$, drag, $D$, and side force, $F_{A y}$, are $[4,14]$ :

$$
L=C_{L} \bar{q} S ; \quad F_{A y}=C_{y} \bar{q} S ; \quad D=C_{D} \bar{q} S
$$

where

$$
\begin{aligned}
& C_{L}= C_{L_{0}}+C_{L_{\alpha}} \alpha+C_{L_{i_{h}}} i_{h}+C_{L_{\delta e}} \delta_{e}+C_{L_{\dot{\alpha}}} \frac{\dot{\alpha} \bar{c}}{2 V_{\mathrm{air}, T}}+C_{L_{q}} \frac{Q \bar{c}}{2 V_{\mathrm{air}, T}} ; \\
& C_{y}=C_{y_{0}}+C_{y_{\beta} \beta} \beta+C_{y_{\delta a}} \delta_{a}+C_{y_{\delta r}} \delta_{r} \quad+C_{y_{\dot{\beta}}} \frac{\beta}{2 V_{\mathrm{air}, T}}+C_{y_{p}} \frac{p \bar{c}}{2 V_{\mathrm{air}, T}}+C_{y_{r}} \frac{r \bar{b}}{2 V_{\mathrm{air}, T}} ; \\
& C_{D}=\bar{C}_{D_{0}}+\frac{C_{L}^{2}}{\pi A e} .
\end{aligned}
$$

Then, the aerodynamic force components along the body axes are

$$
F_{A x}=L \sin \alpha-D \cos \alpha ; \quad F_{A z}=-D \sin \alpha-L \cos \alpha .
$$

Similarly, aerodynamic roll moment, $L_{A}$, pitch moment, $M_{A}$, and yaw moment, $N_{A}$, can be written as

$$
L_{A}=C_{l} \bar{q} S \bar{b} ; \quad M_{A}=C_{m} \bar{q} S \bar{c} ; \quad N_{A}=C_{n} \bar{q} S \bar{b}
$$

where $[4,14]$

$$
\begin{aligned}
& C_{l}=C_{l_{0}}+C_{l_{\beta}} \beta+C_{l_{\delta a}} \delta_{a}+C_{l_{\delta r}} \delta_{r} \\
&+C_{l_{\dot{\beta}}} \frac{\dot{\beta} \bar{b}}{2 V_{\mathrm{air}, T}}+C_{l_{p}} \frac{p \bar{b}}{2 V_{\mathrm{air}, T}}+C_{l_{r}} \frac{r \bar{b}}{2 V_{\mathrm{air}, T}} \\
& C_{m}=C_{m_{0}}+C_{m_{\alpha}} \alpha+C_{m_{i_{h}}} i_{h}+C_{m_{\delta e}} \delta_{e}+C_{m_{\dot{\alpha}}} \frac{\dot{\alpha} \bar{c}}{2 V_{\mathrm{air}, T}}+C_{m_{q}} \frac{Q \bar{c}}{2 V_{\mathrm{air}, T}} \\
& C_{n}=C_{n_{0}}+C_{n_{\beta} \beta} \beta+C_{n_{\delta a}} \delta_{a}+C_{n_{\delta r}} \delta_{r} \\
&+C_{n_{\dot{\beta}}} \frac{\dot{\beta} \bar{b}}{2 V_{\mathrm{air}, T}}+C_{n_{p}} \frac{p \bar{b}}{2 V_{\mathrm{air}, T}}+C_{n_{r}} \frac{r \bar{b}}{2 V_{\mathrm{air}, T}}
\end{aligned}
$$


Thrust force of turbofan engine depends on the sea level maximum thrust $\left(T_{\max , \mathrm{sl}}\right)$, the density ratio $(\sigma)$, the throttle setting $\left(\delta_{T i}\right)$, and the placement of the engine $\left(\varphi_{T i}, \psi_{T i}\right)[4,14]$ :

$$
\begin{aligned}
& \left.T_{x i}=T_{\max , \mathrm{sl}} \sigma \delta_{T i} \cos \varphi_{T i} \cos \psi_{T i}\right] \\
& T_{y i}=T_{\max , \mathrm{sl}} \sigma \delta_{T i} \cos \varphi_{T i} \sin \psi_{T i} \\
& T_{z i}=-T_{\max , \mathrm{sl}} \sigma \delta_{T i} \sin \varphi_{T i}
\end{aligned}
$$

Total thrust force is the sum of thrusts of $n$ engines:

$$
F_{T x}=\sum_{i}^{n} T_{x i} ; \quad F_{T y}=\sum_{i}^{n} T_{y i} ; \quad F_{T z}=\sum_{i}^{n} T_{z i} .
$$

Once the engine is shutdown or become inoperative due to a failure, the fans at the inlet of engine start to produce an extra amount of drag force which is known as wind milling drag and proportional to engine inlet area $A_{\text {eng }}$ and dynamic pressure. Modeling of this force is highly important while dealing with engine failures [15]:

$$
D_{i}=0.3 A_{\text {eng }} \bar{q} .
$$

Moments generated by the engines are found by the product of thrust forces and moment arms at each axis. These moment arms $\left(x_{T i}, y_{T i}, z_{T i}\right)$ are the engine locations with respect to the center of gravity (c.g.):

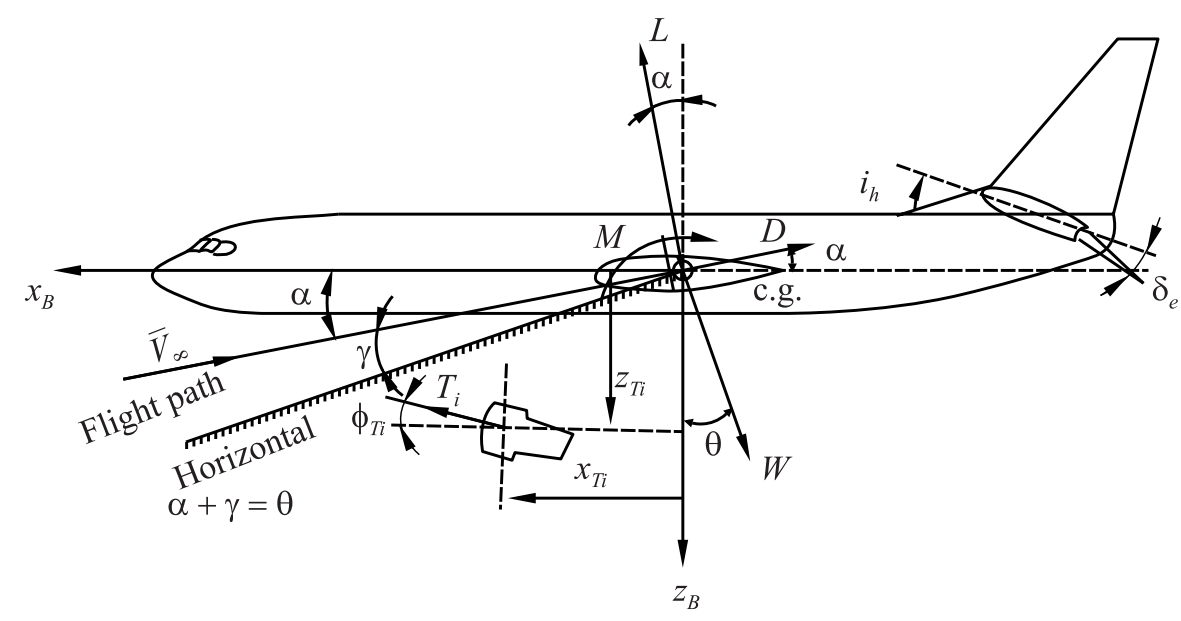

Figure 1 Body axes and the forces acting on the airplane in $x_{B}-z_{B}$ plane: $F_{A x}$ $=L \sin \alpha-D \cos \alpha ; F_{A z}=-L \cos \alpha-D \sin \alpha ; i_{h}, \delta_{e}, i_{c}, \delta_{c}$, and $\phi_{T}$ are positive as shown 


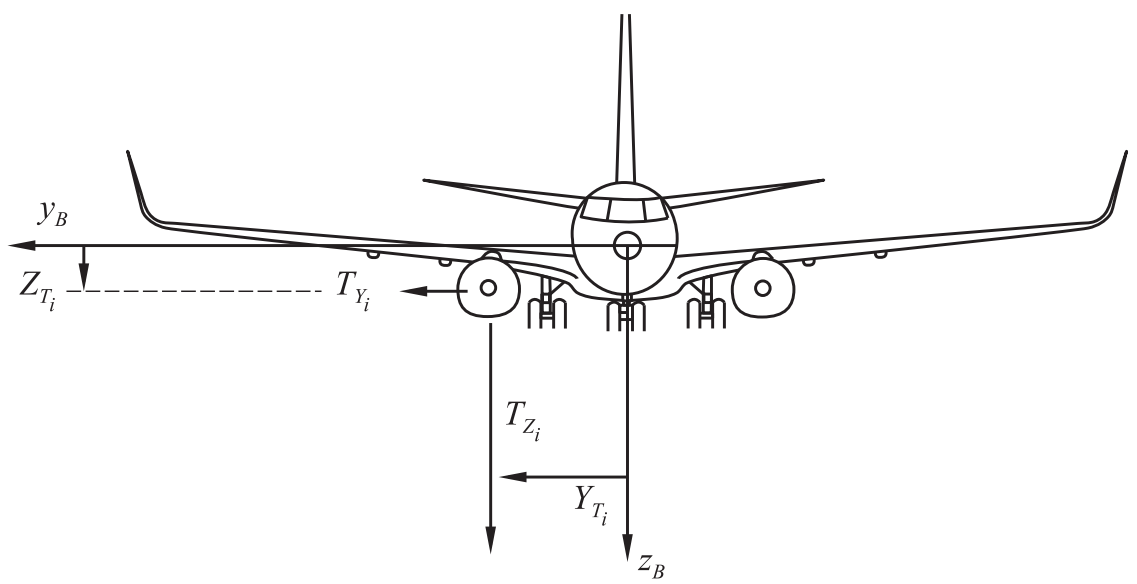

Figure 2 Thrust forces and moment arms in $y_{B}-z_{B}$ plane: $T_{Y_{i}}=T_{i} \cos \Phi_{T} \sin \Psi_{T_{i}}$; $T_{Z_{i}}=-T_{i} \sin \Phi_{T_{i}}$

$$
\left.\begin{array}{rl}
L_{T} & =\sum_{i}^{n}\left(-T_{y i} z_{T i}+T_{z i} y_{T i}\right) ; \\
M_{T} & =\sum_{\substack{n \\
n}}^{n}\left(T_{x i} z_{T i}-T_{z i} x_{T i}\right) ; \\
N_{T} & =\sum_{i}^{n}\left(T_{y i} x_{T i}+T_{x i} y_{T i}\right) .
\end{array}\right\}
$$

The definition of the body axes and the aerodynamic and thrust forces acting on the aircraft are shown in Figs. 1-3 [4, 14].

\section{TRIM ANALYSIS}

A normal cruise flight is an equilibrium state where the net force and moment acting on the aircraft is zero. This is provided by a certain configuration of control surface deflections and engine throttle settings which constitutes trim values of flight mode. In case of a component failure or structural damage, the balance of forces and moments is disrupted and the previous trim values are no longer adequate for equilibrium. Newton-Raphson method is utilized to determine trim values of controls for normal cruise and for cruise with failed components. Different assumptions on states such as sideslip angle or roll angle can be made depending on desired output, before calculating the trim values. For example for a cruise, all unsteady variables $\dot{\alpha}, \dot{\beta}, P, Q, R$, and roll $(\phi)$ and yaw $(\psi)$ angles are set to zero. Other flight parameters such as linear velocities, 


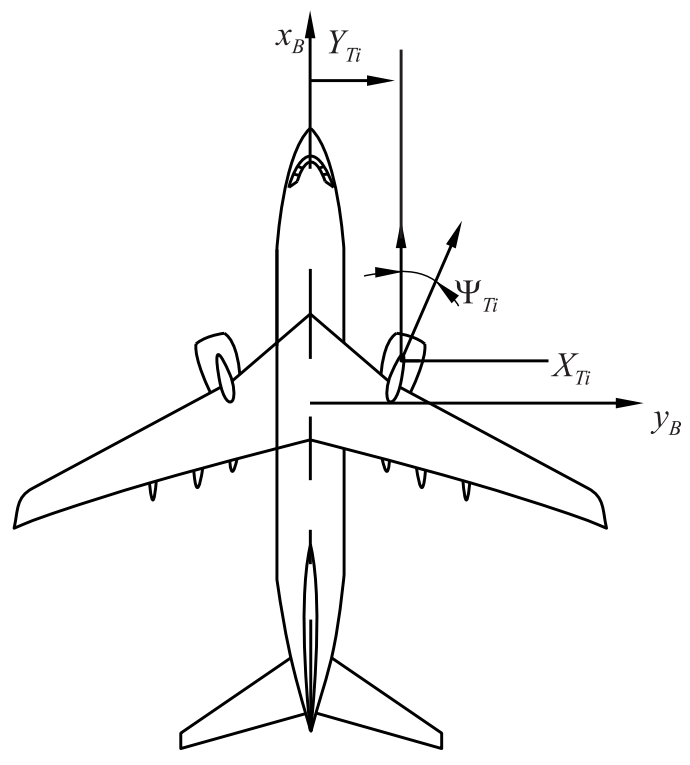

Figure 3 Thrust forces and moment arms in $x_{B}-y_{B}$ plane: $T_{i}=T_{\max , \mathrm{sl}, i} \sigma \delta_{T, i}$; $F_{T_{x i}}=T_{i} \cos \phi_{T_{i}} \cos \psi_{T_{i}} ; F_{T_{y i}}=T_{i} \cos \phi_{T_{i}} \sin \psi_{T_{i}} ; F_{T_{z i}}=-T_{i} \sin \phi_{T_{i}}$

the initial pitch angle, and cruise altitude are assigned to their preferred values. Then, by making initial guesses on the variables trim values, a search algorithm is employed to find the required amounts of control to achieve a balanced flight. Details of the procedures of the trim analysis are explained in [4]. Table 1 shows these necessary amounts of controls.

By inserting the failure into Eqs. (11)-(26), a reference model to damaged aircraft can be obtained. Repeating this trimming routine for failure cases, postfailure trims can be obtained. Then, changing the controls into these values, an open loop mechanism can be tested to stabilize the aircraft.

An engine out (EO) failure can be modeled as removing first engine thrust contribution and introducing wind milling drag. This configuration will suffer from a large amount of yaw moment which will also cause roll motion as equations are strongly coupled. Also, if not compensated, the necessary amount of total thrust will decrease to less than a half and the velocity necessary to produce lift will no longer be available.

Table 1 demonstrates the necessary changes in control surfaces and second engine throttle to achieve the same flying qualities before the EO event. While doing so, it was desired that linear velocity $U$ is unchanged. Also, roll angle is not wanted in postfailure trim.

Another failure type is an actuator related issue. During the normal operation, control surfaces and their actuator may have mechanical problems. In this 
Table 1 Trim values for normal and EO flight conditions

\begin{tabular}{lcclcc}
\hline \multicolumn{1}{c}{ Condition } & $\delta_{t 1}$ & $\delta_{t 2}$ & \multicolumn{1}{c}{$\delta_{e}$} & $\delta_{a}$ & $\delta_{r}$ \\
\hline Normal operation & 0.236145 & 0.236145 & 4.0 & -0.0 & 0.0 \\
First EO & $\mathrm{n} / \mathrm{a}$ & 0.600453 & 4.021679 & 1.479276 & -2.296550 \\
\hline
\end{tabular}

Table 2 Trim values for normal and rudder actuator failure flight conditions

\begin{tabular}{lcclcc}
\hline \multicolumn{1}{c}{ Condition } & $\delta_{t 1}$ & \multicolumn{1}{c}{$\delta_{t 2}$} & \multicolumn{1}{c}{$\delta_{e}$} & $\delta_{a}$ & $\delta_{r}$ \\
\hline Normal operation & 0.236145 & 0.236145 & 4.0 & -0.0 & 0.0 \\
Rudder jammed at 2 & 0.458154 & 0.014137 & 4.002206 & 1.114162 & 2.0 \\
\hline
\end{tabular}

problem, it is assumed that rudder is jammed at $2^{\circ}$. Trim results can be seen in Table 2 .

This approach can be viewed as the actual commands that pilot should apply in case of failures. This kind of action requires a very sharp pilot interference which is practically not possible. There may be over or under reaction as human factor is included. In addition, failures and damages can occur very fast and pilot reaction may be late. A little amount of delay in pilot's response would result in high amount of forces and moments to be generated and to put the aircraft into irreversible positions. For this reason, an autopilot function for detecting and compensating failures can be used.

\section{LINEAR MODEL}

It can be observed from Eqs. (1)-(10) that the aircraft is a naturally nonlinear plant having strong couplings between its modes. To be able to use the linear control design techniques, the plant (aircraft dynamics) should be linearized around trim points of the aircraft. Either numerical or algebraic techniques can be used for this purpose. Nonlinear flight dynamics equations are linearized by small perturbations approximation [16], and the longitudinal and lateral/directional equations are expressed by the dimensioned derivatives

$$
x_{\mathrm{lon}}=\left[\begin{array}{lll}
u w & w & q
\end{array}\right]^{\mathrm{T}} ; u_{\mathrm{lon}}=\left[\begin{array}{lll}
\delta_{t 1} & \delta_{t 2} & \delta_{e}
\end{array}\right]^{\mathrm{T}} ; \dot{x}_{\mathrm{lon}}=A_{\mathrm{lon}} x_{\mathrm{lon}}+B_{\mathrm{lon}} u_{\mathrm{lon}}
$$

where the state variables are the body velocities on $x$ and $z$ axis, pitch rate, and Euler angle around the $y$ axis; and control variables are the engine throttle settings $\delta_{t 1}$ and $\delta_{t 2}$ and the elevator deflection $\delta_{e}$. Similarly, for the lateral part, the state and control vector is selected as

$$
x_{\text {lat }}=\left[\begin{array}{llll}
\nu & p & \phi & \psi
\end{array}\right]^{\mathrm{T}} ; u_{\text {lat }}=\left[\begin{array}{llll}
\delta_{t 1} & \delta_{t 2} & \delta_{a} & \delta_{r}
\end{array}\right]^{\mathrm{T}} ; \dot{x}_{\text {lat }}=A_{\text {lat }} x_{\text {lat }}+B_{\text {lat }} u_{\text {lat }} .
$$




\section{CONTROLLER DESIGN}

\subsection{Engine Out Controller Design}

Using these linear relations, an optimal regulator can be designed to keep the state variables at their prefailure trim values. Throughout the design, it is assumed that all states are readily measurable and control surface actuators have no time constant. In case of an engine failure, system matrices $A_{\text {lon }}$ and $\mathrm{A}_{\text {lat }}$ are not altered; however, control matrices $\mathrm{B}_{\text {lon }}$ and $\mathrm{B}_{\text {lat }}$ are subjected to change. For the linear quadratic control design, conventional cost function $J$ is minimized [16]:

$$
J=\int_{0}^{\infty}\left(x^{\mathrm{T}} Q x+u^{\mathrm{T}} R u\right) d t
$$

where $R$ matrix determines the amount of control power to be used and it is taken as the identity matrix for both longitudinal and lateral controller designs; and $Q$ matrix determines the maximum variation of system states from the equilibrium, it is chosen by some experiments to give a satisfactory time response. Using the optimal state feedback theory, necessary optimal gains that will assure this time response can be obtained.

When the first engine (left engine) goes inoperative, control variable $\delta_{t 1}$ has no effect on the equations. Therefore, for longitudinal control, only the second engine throttle $\delta_{t 2}$ and the elevator deflection $\delta_{e}$ are used. For the lateral subsystem control, aileron and rudder deflections are used:

$$
u_{\mathrm{lon}}=\left[\begin{array}{c}
\delta_{t 2} \\
\delta_{e}
\end{array}\right]=-K_{\mathrm{lon}} x_{\mathrm{lon}} ; \quad u_{\mathrm{lat}}=\left[\begin{array}{c}
\delta_{a} \\
\delta_{r}
\end{array}\right]=-K_{\mathrm{lat}} x_{\mathrm{lat}}
$$

Optimal Kalman gains $K_{\text {lon }}$ and $K_{\text {lat }}$ are calculated via solving the Algebraic Riccati equation:

$$
0=A^{\mathrm{T}} P+P A+Q-P B R^{-1} B^{\mathrm{T}} P
$$

so, the gain:

$$
K=R^{-1} B^{\mathrm{T}} P .
$$

MATLAB ${ }^{\circledR}$ control toolbox is used for solving these equations to obtain longitudinal and lateral gains. The important part is integrating this controller to nonlinear aircraft model as an EO case is a highly nonlinear phenomenon that has both lateral and longitudinal effects on the body.

For this reason, the earlier SIMULINK ${ }^{\circledR}$ model that was used to simulate the aircraft motion was updated. In previous simulation, an open loop mechanism was used to simulate a very sharp pilot movement to put the aircraft into a trimmed state such that all variables had the correct necessary values to 
continue the cruise safely with an EO. However, for this simulation, the pilot is assumed to be unresponsive to the failure. When the engine failure occurs, the sensor unit connected to the system produces an EO signal that activates the automatic control routine. Measuring the current changes in states, the autopilot attempts to minimize these variations by feed-backing the loop. In the following subsection, the results of autopiloted simulation are compared to previous open loop and no control cases.

\subsection{Rudder Jam Controller Design}

In this scenario, the rudder is no longer available for feedback control. Different from the previous part, both engines are healthy; so, a thrust difference scheme can be an alternative to rudder for yaw control. For this reason, this time throttles are not only in longitudinal part. Therefore, two decoupled parts must be connected. Elevator and ailerons are also included. Equation (27) is resolved for new control and system matrices:

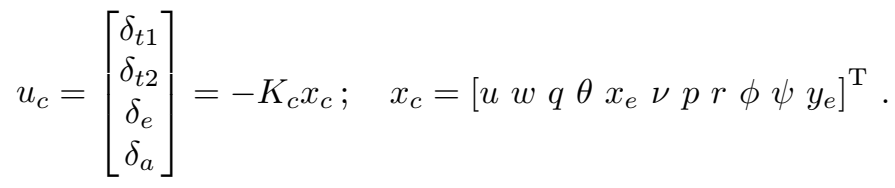

\section{SIMULATION RESULTS}

From the simulations, it was observed that no matter how sharply the pilot applies commands, some portions of moments and forces were still active and the aircraft was under control of a side force which causes a large $y$-axis displacement growing with time. State, control, and other variables depending on them are compared.

\subsection{Engine Out Simulation}

The results obtained from the three cases of simulation for EO have been compared. No control option is the case where no control action is taken after the failure. Figure 4 shows that open loop and autopilot angle of attack and pitch angle are almost the same, except for some small variations. This is due to the fact that engine thrust is mainly effective on angle of attack. Also, from Fig. 5, it can be observed that changes of longitudinal controls are almost identical for open and close loop schemes.

It can be observed from Fig. 4 that sideslip angles are different for these two cases. When open loop control deflection values were calculated, it was asserted 
into the equations to make the roll angle to be zero. This was one of the reasons of undesired lateral displacement.

Since the variation in vertical component of the body velocity kept small, the altitude is almost identical. There is only a minor variation that is compensated in a short time. The open loop control takes care of many states well, but attempting to keep the roll angle at zero velocity on $y$ axis and side slipping
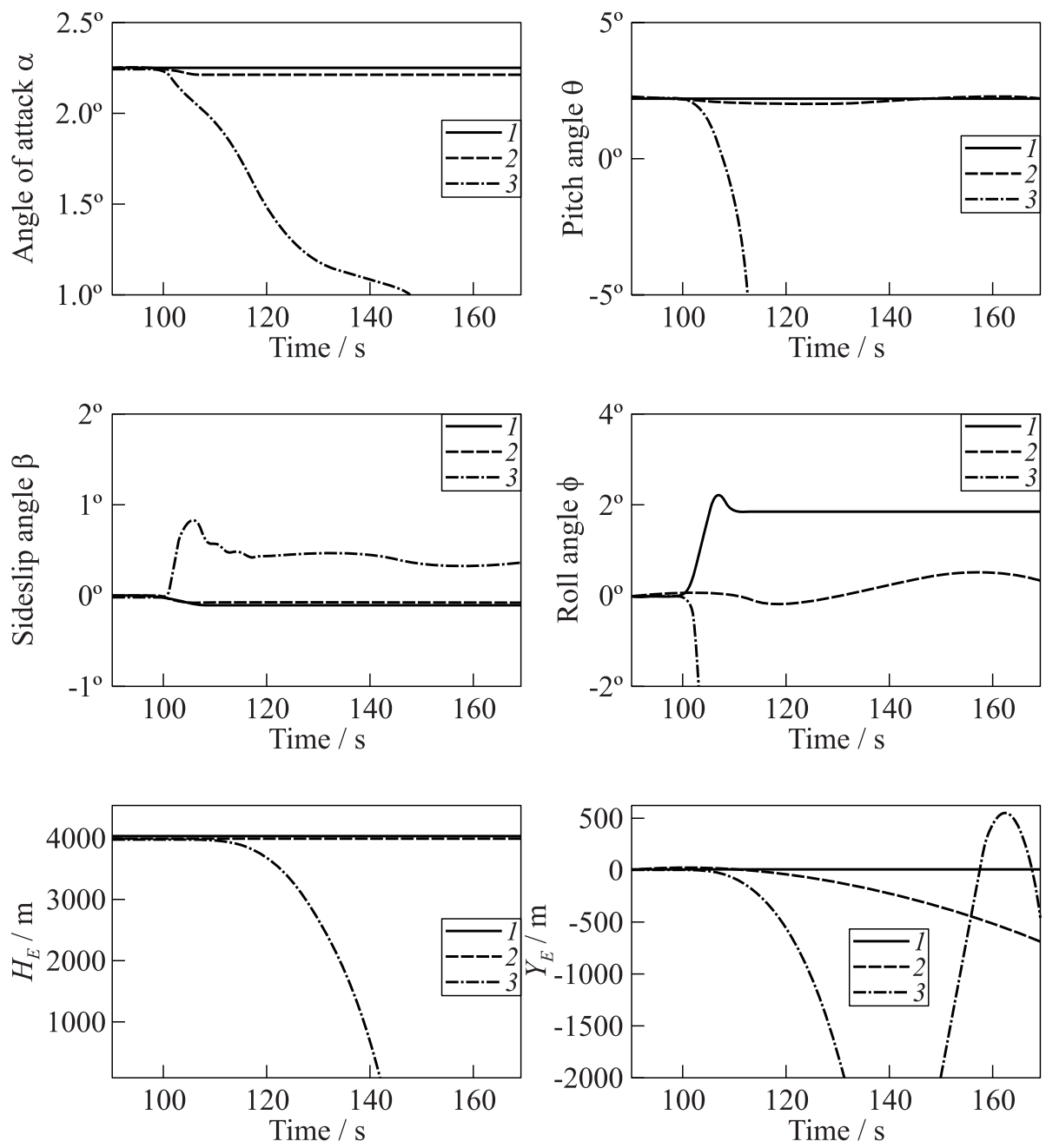

Figure 4 Comparison of angle of attack, pitch angle, sideslip and roll angle, altitude, and lateral displacement variations for EO case: 1 - closed loop; 2 - open loop; and 3 - no control 
cannot be diminished. Autopilot allows the aircraft to roll that is a disturbance to passengers and crew, as a result lacking the side force and lateral displacement.

In Fig. 5, the change of control deflections is given. For the longitudinal case, both the elevator deflections $\delta_{e}$ and second engine thrust $\delta_{t 2}$ have very close results.
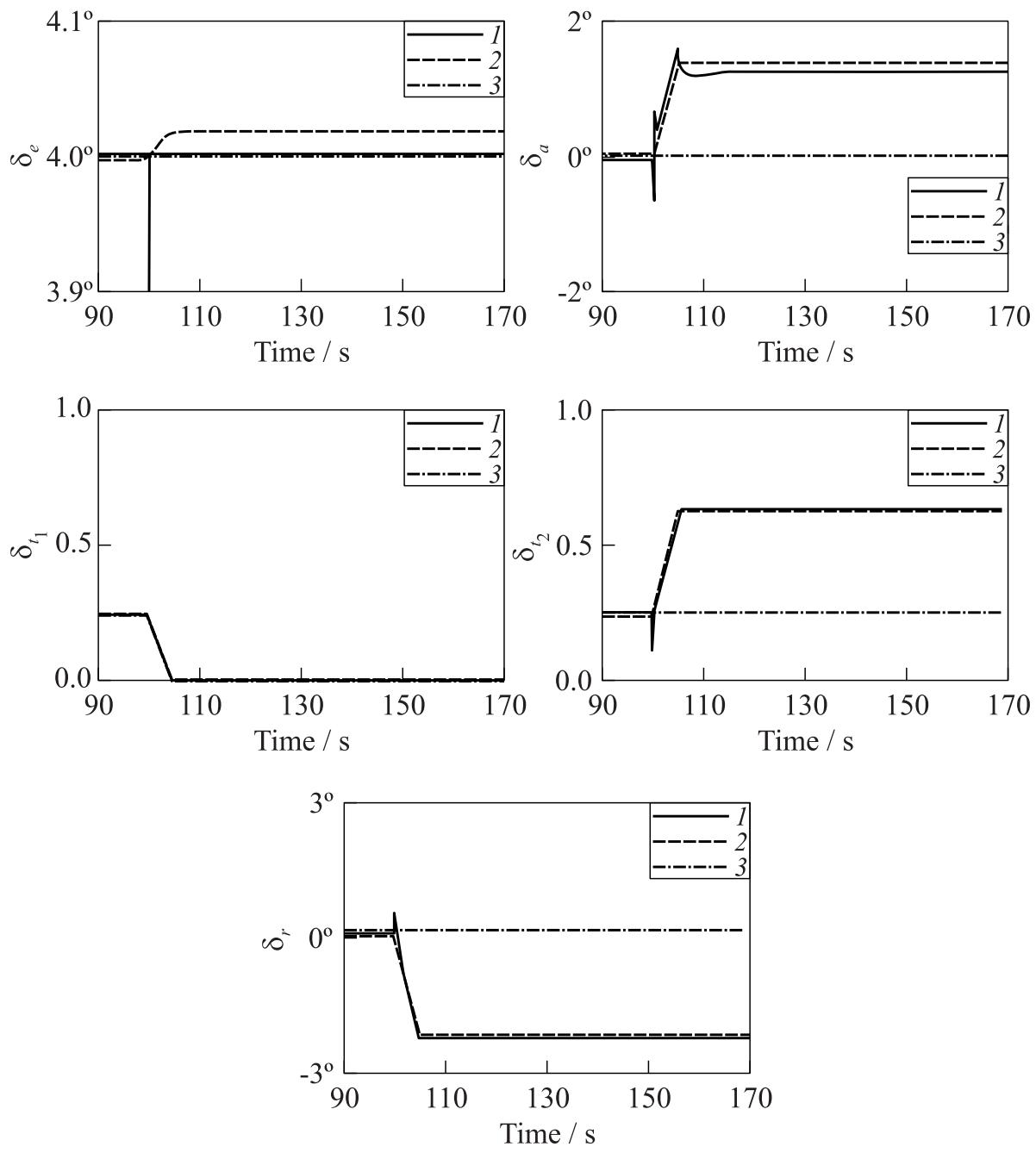

Figure 5 Comparison of control deflections $\delta_{e}, \delta_{a}, \delta_{t 1}, \delta_{t 2}$, and $\delta_{r}$ for EO case: $1-$ closed loop; 2 - open loop; and 3 - no control 


\subsection{Rudder Jammed Simulation}

When the rudder actuator jams at a certain degree, it causes a yaw moment and a side force. Despite $2^{\circ}$ is small, if no control action compensates these yaw effects then it leads aircraft to fall. Figure 6 depicts the sideslip angle and the
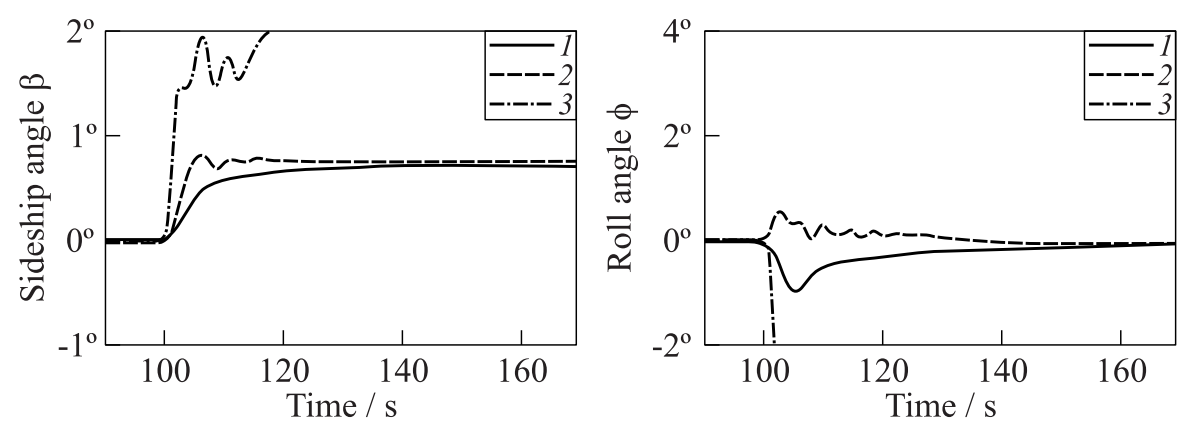

(a)
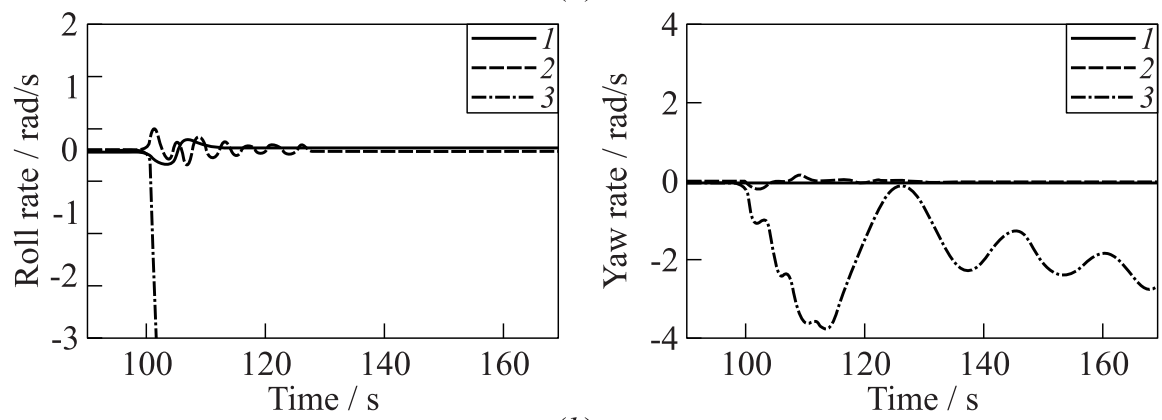

(b)
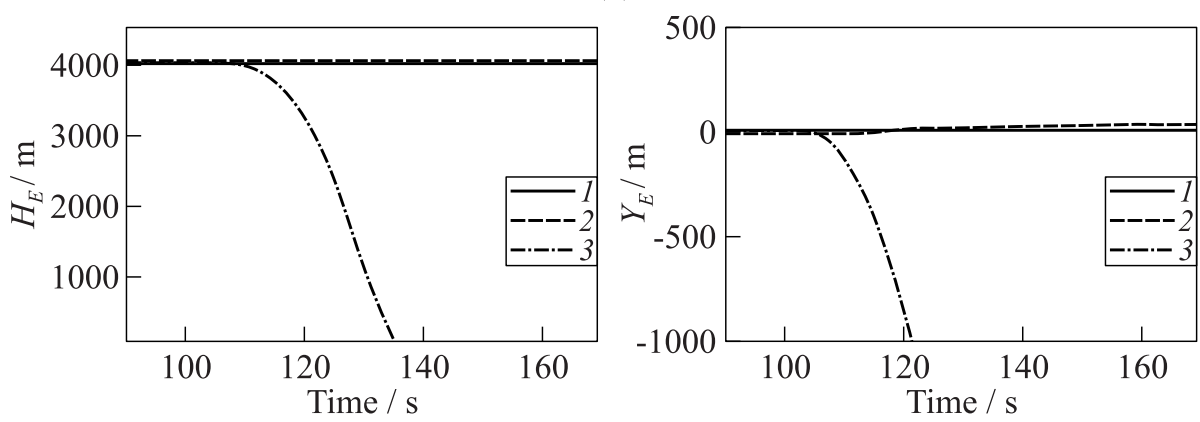

(c)

Figure 6 Comparison of sideslip and roll angles $(a)$, roll and yaw rates (b), and altitude and lateral displacement variations $(c)$ for rudder jam case: 1 - closed loop; 2 - open loop; and 3 - no control 
roll angle variations. Open loop and closed loop Linear Quadratic Regulator (LQR) results are very similar to each other. Roll and yaw rates are increased for a short time when the failure occurs, but quickly they settle to zero. Closed loop is smoother as it has the error feedback continuously.

The altitude and lateral displacement shown in Fig. 6 states that the failure effects only very small amount of variation with open loop and closed loop con-
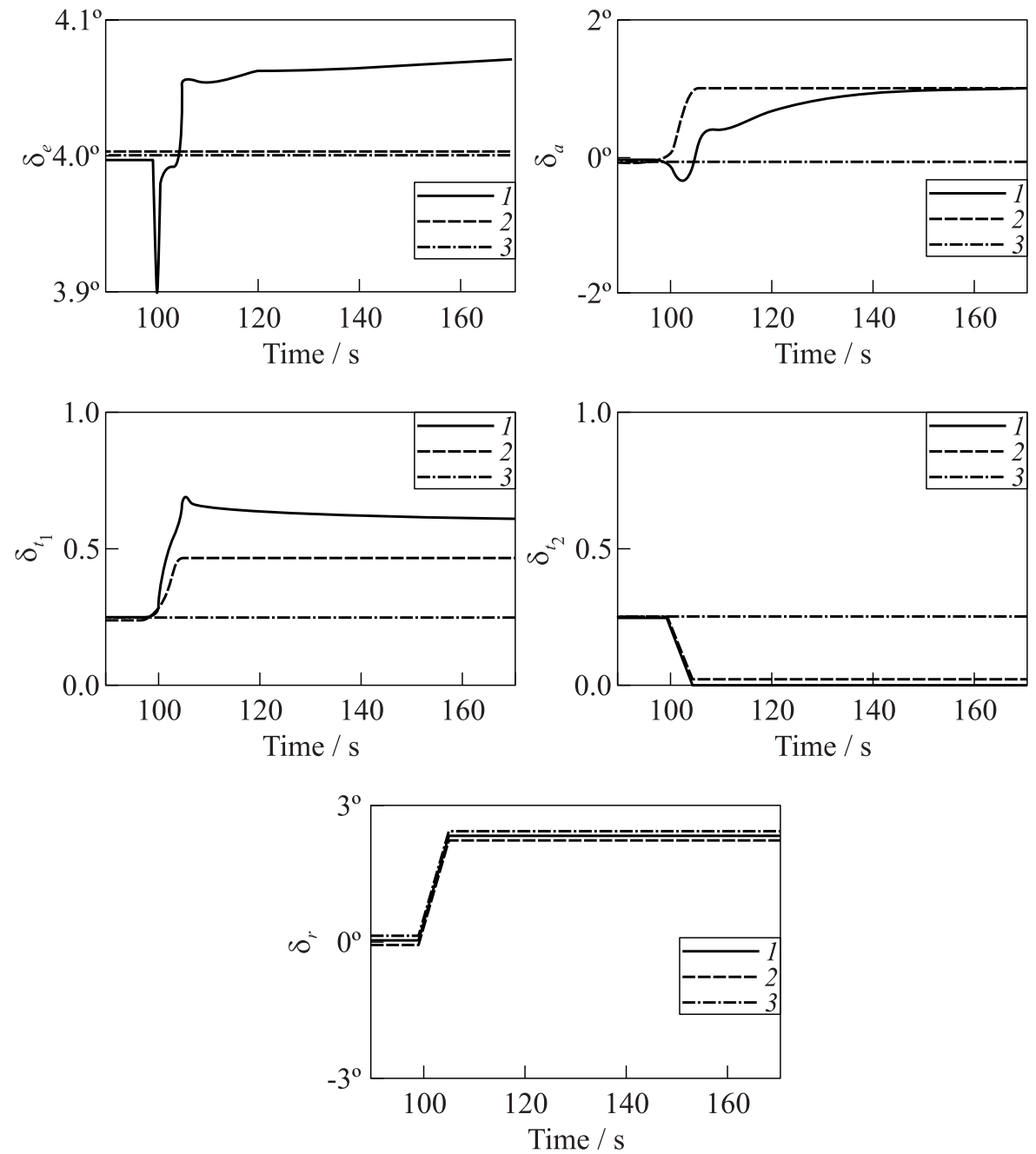

Figure 7 Comparison of control deflections $\delta_{e}, \delta_{a}, \delta_{t 1}, \delta_{t 2}$, and $\delta_{r}$ for rudder jam case: 1 - closed loop; 2 - open loop; and 3 - no control 
trollers. For the altitude change, both have the same time domain specifications. However, for the lateral displacement, the open loop variation is larger than the closed loop variation and keeps slowly increasing.

In Fig. 7, the change of control deflections is given for the rudder jam problem. The first engine throttle value increases to about its double value, while the second engine throttle decreases to almost zero. Elevator and aileron deflections are more similar than other oscillations. This failure is mainly controlled by thrust difference and is an example to the topic called Thrust Controlled Aircraft.

\section{CONCLUDING REMARKS}

In this study, the stabilization of a twin engine transport aircraft is studied after failures "one engine full thrust loss" and "rudder actuator jammed." Two approaches are tested for each case. The first approach (open loop) is based on obtaining the postfailure trim conditions and varying the controls to new trim values after the failure.

In the former open loop approach, the roll angle $(\phi)$ was kept constant, thus, the postfailure sideslip angle $(\beta)$ was different from the prefailure value. Open loop control was successful to eliminate sudden and large changes in trajectory. The latter approach (closed loop) is aimed at minimizing the changes in aircraft attitude states by utilizing the optimal control theory. Linear Quadratic Regulator method is applied. Different weighting coefficients are assigned to different states. The highest weighting coefficient was assigned to lateral displacement. As a result, the aircraft made a small roll of about $2^{\circ}$ but there was a little variation in side slip angle and aircraft continued its flight path without any observable trajectory change in vertical and side directions. For rudder jammed case, open loop analysis and closed loop control design implemented similarly. The difference of two engines' thrust force was utilized to compensate the yaw effect generated by the failure. Instead of longitudinal and lateral states, one combined state is used to solve Riccati equation.

This study is the first step to a detailed flight dynamics analysis and control synthesis problem. In future work, more detailed failures altering the model to a great extent can be covered. For these kinds of failures, techniques such as State-dependent Riccati equation and different control allocation methods such as Pseudoinverse and Daisy chaining may be utilized as both the model and the controller parameters are subject to change.

\section{ACKNOWLEDGMENTS}

This research is supported by the Scientific and Technical Research Council of Turkey (TUBITAK) through Project No. 108M470. 


\section{REFERENCES}

1. McLean, D. 1997. An automatic engine-out recovery system. Aviation Safety Conference (International) IASC 97 Proceedings. Amsterdam. 763-76.

2. Magni, C.F., S. Bennani, and J. Terlouw. 1997. Robust flight control. London: Springer.

3. Kordt, M., and J. Ackermann. 2003. Aircraft load alleviation by robust yaw-lateral decoupling. Automatica 39(11): 1885-91.

4. Özdemir, U., and M. S. Kavsaoğlu. 2010. Simulation and control of fixed-wing aircraft after a major component loss. 48th AIAA Aerospace Sciences Meeting. Orlando, Florida, USA.

5. Shtessel, Y. B. 1997. Nonlinear output tracking in conventional and dynamic sliding manifolds. IEEE Trans. Automatic Control 42(9):1282-86.

6. Hess, R. A., S. R. Wells, and T. K. Vettelf. 2002. MIMO sliding mode control as an alternative to reconfigurable flight control designs. American Control Conference Proceedings. Anchorage, Alaska, USA.

7. Boskovic, J. D., Li Sai-Ming, and R.K. Mehra. 2000. Reconfigurable flight control design using multiple switching controllers and on-line estimation of damagerelated parameters. 2000 IEEE Conference (International) on Control Applications Proceedings. Anchorage, Alaska, USA.

8. Bodson, M., and J. Groszkiewicz. 1994. Multivariable adaptive algorithms for reconfigurable flight control. 33rd IEEE Conference on Decision and Control Proceedings.

9. Liu, Y., G. Tao, and S. M. Joshi. 2006. Direct adaptive actuator failure compensation techniques with applications to aircraft flight control systems. AIAA Paper No. 2006-6554.

10. Mack S., G. Tao, and J. O. Burkholder. 2010. An adaptive detection scheme for aircraft aerodynamic system damage. 2010 American Control Conference. Baltimore, MD, USA.

11. Yechout, T. R., S. L. Morris, D. E. Bossert, and W. F. Hallgren. 2003. Introduction to aircraft flight mechanics: Performance, static stability, dynamic stability and classical feedback. Reston, VA: AIAA.

12. Özdemir, U., and M.S. Kavsaoğlu. 2008. Linear and nonlinear simulations of aircraft dynamics using body axis system. Int. J. Aircraft Engng. Aerospace Technol. 80(6):638-48.

13. DAR Corporation. 2004. Advanced Aircraft Analysis (AAA). Version 2.5 Software Package User's Manual.

14. Roskam, J. 2001. Airplane flight dynamics and automatic flight controls. Parts I and II. DAR Corp.

15. Raymer, D. P. 1992. Aircraft design: A conceptual approach. 2nd ed. AIAA education ser.

16. Stevens, B. L., and L. L. Frank. 2003. Aircraft control and simulation. 2nd ed. John Wiley. 